\title{
Load transfer in phase transforming matrix-nanowire composite revealing the significant load carrying capacity of the nanowires
}

Cun $\mathrm{Yu}^{\mathrm{a}}$, Zhenyang Liu ${ }^{\mathrm{a}, \mathrm{b}}$, Yinong Liu ${ }^{\mathrm{c} *}$, Yang Shao ${ }^{\mathrm{a}}$, Yang Ren ${ }^{\mathrm{d}}$, Lishan Cui ${ }^{\mathrm{a} *}$

${ }^{a}$ Department of Materials Science and Engineering, China University of Petroleum-Beijing, Beijing 102249 China

${ }^{b}$ Beijing General Research Institute of Mining and Metallurgy, Beijing 100160, China

'School of Mechanical and Chemical Engineering, The University of Western Australia, Crawley, WA 6009, Australia

${ }^{d} X$-ray Science Division, Argon National Laboratory, Argonne, Illinois 60439, USA

*Author to whom correspondence should be addressed. E-mail: lscui@cup.edu.cn (L. Cui), Tel.: +86 10 89733975; email: yinong.liu@uwa.edu.au (Y. Liu), Tel.: +61 864883132

\begin{abstract}
:
This paper reports a study of an in-situ composite of NiTi matrix and aligned Nb nanowires. The design strategy of the composite was to further explore the load carrying capacity of the nanowire reinforcements in composite. This composite system offered a unique condition of load sharing between the two components in which the NiTi matrix deforms via discrete (discontinuous), instantaneous and intrinsic lattice distortion through stress-induced martensitic transformation (SIMT) and the Nb nanowires deform via elastic deformation. This study investigated the mechanism of load sharing between the embedded Nb nanowires and the NiTi matrix by means of in-situ synchrotron diffraction analysis. It was found that significant load transfer from the matrix to the nanowires occurred when the NiTi matrix underwent stress-induced B2-B19' martensitic transformation and the nanowires deformed largely by elastic deformation. The embedded $\mathrm{Nb}$ nanowires, with a volume fraction of $25 \%$, were revealed capable of carrying at maximally $70 \%$ of the applied load at the completion of SIMT of NiTi matrix, and were capable of carrying more than $55 \%$ of the applied load at the terminal of deformation.
\end{abstract}

Keywords: Composite, NiTi, shape memory alloy, martensitic transformation, nanowire

\section{Introduction}

Bulk metallic materials generally exhibit strengths well below what may be expected of their theoretical load-bearing capacities, owing to the occurrence of plasticity caused by the activities of structural defects. One clear indicator of such early failure is that elastic strain limits of bulk metallic materials are usually no larger than $1 \%$. Metallic nanowires, on the other hand, have been shown to exhibit ultra large elastic strains and exceptional load carrying capacities. Lu et al. reported an elastic strain of over 5\% in [111]-oriented single crystal gold nanowires, as measured by in-situ tensile test inside a transmission electron 
microscope (TEM) [1]. Richter et al. [2] observed ultra large elastic strains of $\mathrm{Cu}$ nanowires approaching $4 \%$ in scanning electron microscope (SEM) and Yue et al. [3] made a similar observation of $7.2 \%$ elastic strain in TEM during in-situ tensile deformation. Wang et al. reported maximum elastic strains of $6 \sim 10 \%$ in $\alpha-\mathrm{Al}_{2} \mathrm{O}_{3}$ nanowires fractured in bending mode [4]. Similarly, Ngo et al. measured an ultimate strength of $17 \mathrm{GPa}$ in Germanium nanowires [5], implying an elastic strain limit of $12 \%$. Such extraordinary properties of nanowires have motivated much effort to create applicable ultra-strength engineering materials.

In order to harness the exceptionally high strengths and large elasticity of the nanowires, many attempts have been made to design and fabricate nanowire-reinforced composites [6-9]. Raabe et al. reported a Cu-Ag-Nb ternary filamentary composite, which exhibited a yield strength exceeding $1800 \mathrm{MPa}$ [9]. Thilly et al. and Ohsaki et al. reported Cu-Nb and cold worked $\mathrm{Cu}-\mathrm{Ag}$ filamentary composite with maximum tensile strengths of $1150 \mathrm{MPa}$ and $1750 \mathrm{MPa}$, respectively $[8,10]$. The strengths of these composites are noticeably several times higher than the tensile strengths of each of the components in their bulk forms, apparently due to the contribution of the nanowires in the composites. For example, the elastic strain of $\mathrm{Nb}$ nanowires embedded in a copper matrix is found to be about $1.9 \%$ [11-13], 9 times larger than that of the bulk $\mathrm{Nb}$, which yields typically after $0.2 \%$ of elastic deformation. However, these improvements are still well below what may be expected of these metallic nanowires. This inability to achieve large elastic strains, thus load bearing contribution, of nanowires in bulk composites has been referred to as the "valley of death" in composite design [14-16].

Recently, a NiTi matrix-Nb nanowire composite with exceptional mechanical properties was reported [17]. Its ultra large quasi-linear elastic strain of $\sim 6 \%$ and high yield strength of $\sim 1.65$ GPa are attributed to the exceptional load bearing capacity of the Nb nanowires and the efficient load transfer from the NiTi matrix to the embedded Nb nanowires [17]. This composite presents a novel metallurgical system in which the NiTi undergoes a lattice distortion associated with a martensitic transformation during deformation, as opposed to plastic deformation via dislocation slip. The embedded Nb nanowires achieved ultra large elastic strains up to $6.5 \%$ [17], presenting a breakthrough to the deadlock of limited elastic strains, hence strength contribution, of nanowires in conventional metal matrices.

The design strategy of the NiTi-Nb composite have yet to be well interpreted before the underlying load sharing mechanism between the NiTi matrix and the Nb nanowires is fully understood. Upon loading, following an initial (small) elastic deformation, the NiTi matrix deforms via stress-induced martensitic transformation (SIMT) during which the matrix exhibits complete compliance (literarily zero modulus of elasticity) over a large range of strain with little stress increase. The nanowires, on the other hand, deform elastically following the Hooke's law until it reaches yield strength. This raises questions on how the load is transferred from the matrix to the nanowires during SIMT and to what extend the nanowires may contribute to the total load carrying capacity of the composite. This work was conducted to study the load sharing mechanism between the $\mathrm{Nb}$ nanowires and the $\mathrm{NiTi}$ matrix in the NiTi-Nb composite by means of in-situ synchrotron X-ray diffraction analysis 
during deformation.

\section{Experimental procedure}

An ingot with a nominal composition of $\mathrm{Ti}_{42} \mathrm{Ni}_{38} \mathrm{Nb}_{20}$ (at. \%) was prepared by means of vacuum induction melting. The ingot of $15 \mathrm{~mm}$ in diameter and $25 \mathrm{~mm}$ in length was annealed at $1153 \mathrm{~K}$ for $10.8 \mathrm{ks}$. The homogenized ingot was hot forged at $1123 \mathrm{~K}$ and then hot drawn into a wire of $0.9 \mathrm{~mm}$ in diameter. The wire was annealed at $1023 \mathrm{~K}$ for $1.2 \mathrm{ks}$ and then further cold drawn to $0.5 \mathrm{~mm}$ in diameter. Tensile samples of $120 \mathrm{~mm}$ in length were cut from the cold drawn wire and annealed in air at $673 \mathrm{~K}$ for $1.2 \mathrm{ks}$. The samples were deformed in tension at room temperature with a strain rate of $1 \times 10^{-4} \mathrm{~s}^{-1}$, with in-situ X-ray diffraction analysis performed at the 11-ID-C beamline of the Advanced Photon Source facility of the Argonne National Laboratory. The high-energy x-rays with a beam size of $0.4 \times 0.4 \mathrm{~mm}^{2}$ and wavelength of $0.10798 \AA$ were used to obtain two-dimensional (2D) diffraction patterns in the transmission geometry. Each diffraction pattern was collected every $0.25 \%$ of the applied strain and the exposure time was $30 \mathrm{~s}$. It should be noted that the deformation strain was determined by measuring the original gauge length of testing sample and the deformation displacement. The sample to detector distance was $2000 \mathrm{~mm}$. The "raw" data process was performed in FIT2D software[18], and the texture was interpreted by Rietveld fitting embedded in Maud software[19]. SEM observation was carried on a FEI Quanta 200F scanning electron microscope operated at a voltage of $20 \mathrm{kV}$. TEM observation was carried out using an FEI Tecnai G20 transmission electron microscope operated at an accelerating voltage of $200 \mathrm{kV}$.

\section{Results and discussion}

Fig. 1 shows the microstructure of the composite wire. Fig. 1a is an SEM back scattered electron micrograph of the cross-sectional view of the wire sample, in which the white dots are the ends of the $\mathrm{Nb}$ nanowires. Fig. $1 \mathrm{~b}$ is a TEM bright field image of the longitudinal view of the wire, and the inset is the corresponding selected area electron diffraction (SAED) pattern. It is seen that the majority of the $\mathrm{Nb}$ nanowires are $30-100 \mathrm{~nm}$ in diameter with large aspect ratios. They are parallel-aligned along the axial direction of the composite wire. The NiTi matrix is polycrystalline with average grain size of $40-60 \mathrm{~nm}$. The volume fraction of the $\mathrm{Nb}$ nanowires was estimated to be about $25 \%$ based on microstructure image analysis, which is also consistent with the estimation based on the pseudo-binary TiNi-Nb phase diagram [20]. Fig. 1c is a high-energy X-ray 2D diffraction pattern of the composite wire. The pattern can be fully indexed to B2-NiTi and BCC-Nb. The $0^{\circ}$ direction in the diffraction pattern corresponds to the wire radial direction and the $90^{\circ}$ direction corresponds to the wire axial direction. It is also noticeable that the diffraction rings of $\mathrm{Nb}$ are highly discontinuous, and demonstrate strong $<110>$ orientation along the axial direction of the composite wire. 

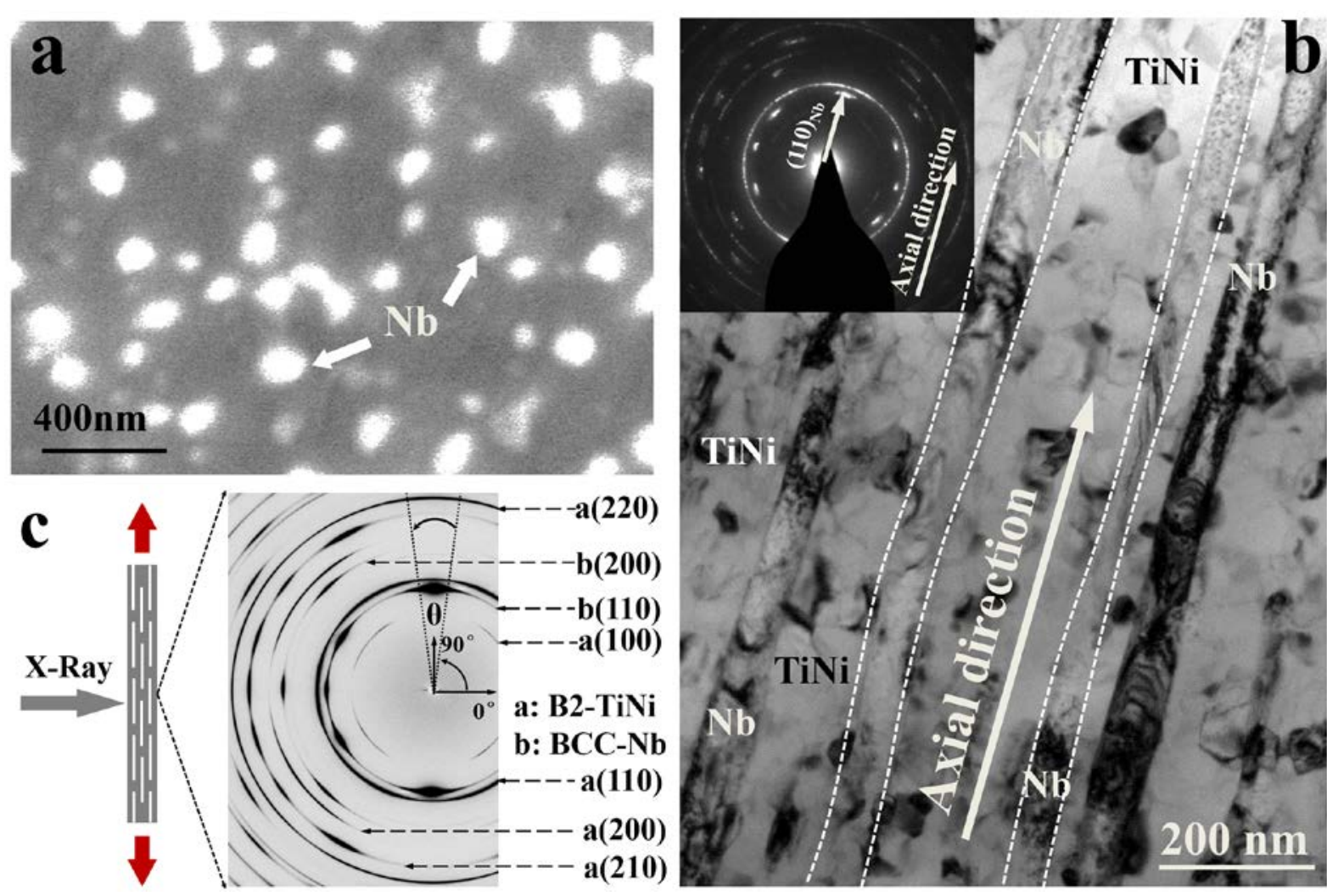

Fig. 1. Microstructure of the NiTi-Nb nanowire composite wire. a: SEM-BSE image of the cross-sectional view of the composite wire; b: TEM micrograph of the longitudinal view of the composite wire; c: schematic of synchrotron X-Ray diffraction analysis and a 2D diffraction pattern of the composite wire.

Fig. 2 shows two inverse pole figures revealing the textures of the $\mathrm{Nb}$ nanowires and the NiTi matrix in the NiTi-Nb composite. Texture intensities are given in multiples of random distribution (m.r.d). Fig. 2a is the inverse pole figure of the $\mathrm{Nb}$ nanowires along the longitudinal direction (LD) and Fig. $2 b$ is the inverse pole figure of the NiTi matrix along the longitudinal direction (LD). It is evident that the $\mathrm{Nb}$ nanowires have a very strong $<110>$ texture along the length direction of the composite wire, whereas the NiTi matrix showed a weak near (111) fiber texture.
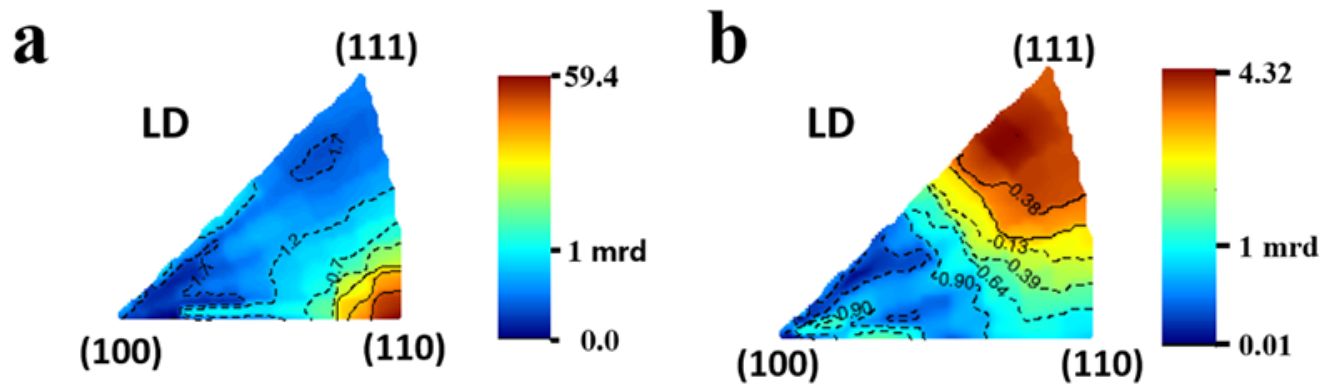

Fig. 2. Inverse pole figures showing the texture of the Nb nanowires and NiTi matrix in the $\mathrm{NiTi}-\mathrm{Nb}$ nanocomposite. a: inverse pole figure of the $\mathrm{Nb}$ nanowires along the length direction of the composite wire; b: inverse pole figure of the NiTi matrix along the length direction of 
the composite wire;

Fig. 3 shows the tensile stress-strain behavior of the composite wire and the corresponding structural evolution as detected by x-ray diffraction. Fig. 3a is the tensile stress-strain curve of the sample. Fig. 3b shows the X-ray diffraction spectra collected within $5^{\circ}$ along the sample's axial direction, as indicated in Fig. 1c. These diffraction patterns represent atomic planes oriented in the direction perpendicular to the axial direction (also the loading direction) of the composite wire. Fig. 3c shows a collection of diffraction patterns obtained by integrating from $0^{\circ}$ to $360^{\circ}$ azimuthal angle of the $2 \mathrm{D}$ patterns. The coloring of diffraction patterns correspond to the three different stages of the deformation, as indicated in both Figs. 3a and c.
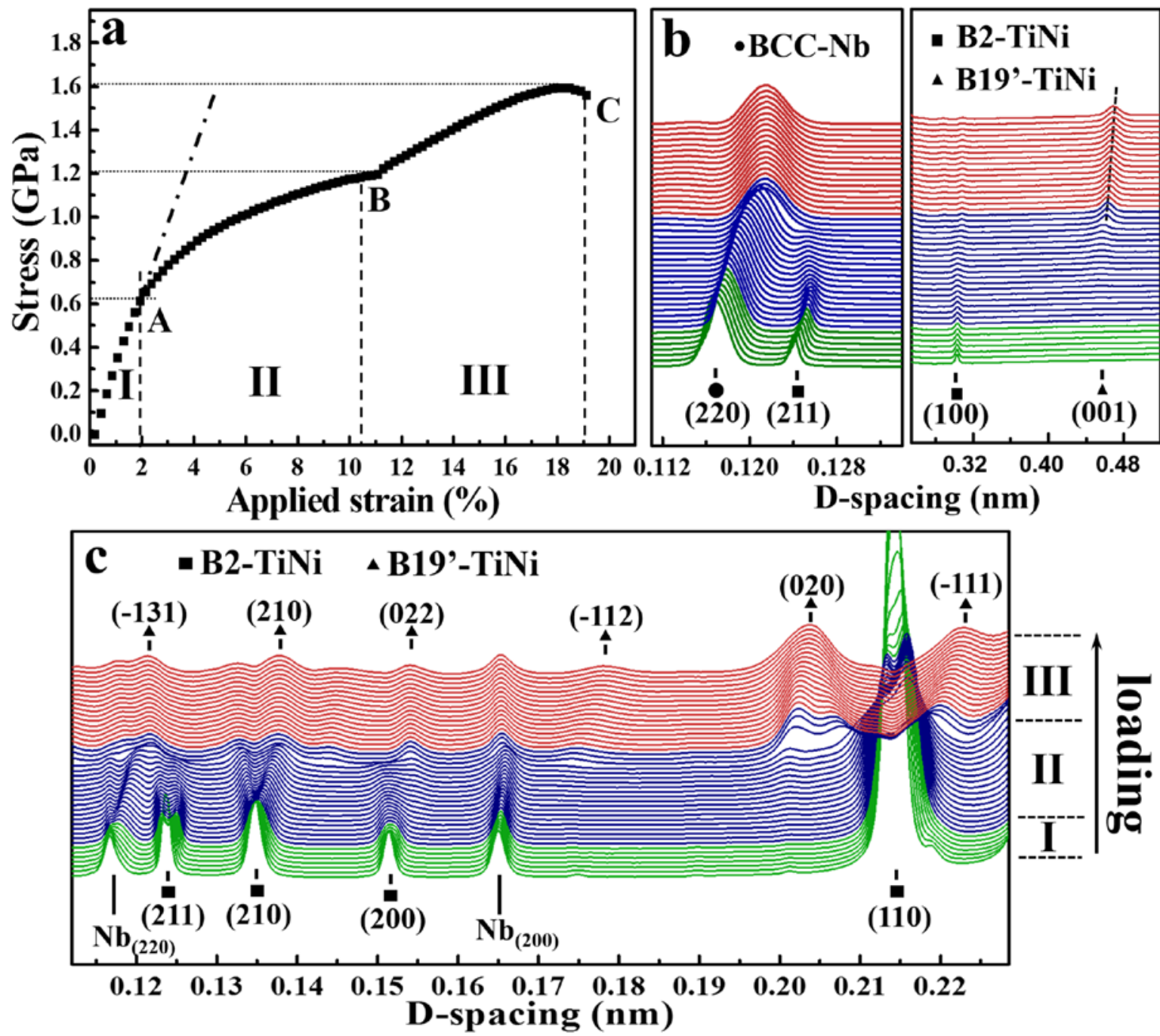

Fig. 3. Tensile deformation behavior of the composite wire. a: tensile stress-strain curve; b: collection of section of $1 \mathrm{D}$ diffraction patterns integrated within $85^{\circ}-95^{\circ}$ azimuthal angle of the $2 \mathrm{D}$ patterns; c: collection of $1 \mathrm{D}$ diffraction patterns integrated within $0-360^{\circ}$ azimuthal angle of the $2 \mathrm{D}$ patterns. 
The deformation process of the sample can be divided into three stages by two turning points upon the applied strain vs. stress curve (Fig. 3a). Stage I is defined as elastic regime, and is characterized by the linear relationship of stress and applied strain. During this stage, both the $\mathrm{Nb}$ nanowires and the B2-NiTi matrix deformed elastically and the load sharing between $\mathrm{NiTi}$ matrix and $\mathrm{Nb}$ nanowires followed the rule of mixture. The (220) $\mathrm{Nb}$ and (211) $)_{\mathrm{B} 2-\mathrm{NiTi}}$ diffraction peaks shifted gradually to higher d-spacing values, as evident in Fig. 2b, demonstrating the lattice dilation of both phases along the axial direction. In stage II the stress increase is slowed down as $\mathrm{NiTi}$ matrix underwent stress induced martensitic transformation. It is seen in Fig. 2C that (211) $)_{\mathrm{B} 2-\mathrm{NiTi}},(210)_{\mathrm{B} 2-\mathrm{NiTi}},(200)_{\mathrm{B} 2-\mathrm{NiTi}}$ and (110) $\mathrm{B} 2-\mathrm{NiTi}$ diffraction peaks all started splitting. By the end of this stage, the intensity of (110) B2-NiTi and (200) B2-NiTi peak decreased significantly whilst those of (022)B19'-NiTi, (020)B19'-NiTi and $(-111)_{\mathrm{B} 19^{\prime}-\mathrm{NiTi}}$ diffraction peaks increased. The splitting of the $\mathrm{B} 2$ peaks on the $1 \mathrm{D}$ diffraction patterns, which were integrated within $0^{\circ}-360^{\circ}$ of the $2 \mathrm{D}$ patterns, is due to the opposite elastic straining on lattice planes along the axial direction and the transverse direction of the sample under uniaxial tension. The diminishing of the B2 peaks and the emergence of the B19' peaks indicate that the $\mathrm{NiTi}$ matrix has undergone stress induced B2 to B19' transformation. The Nb nanowires, however, continued to deform elastically in this stage, as indicated by the continuous shift of $(220)_{\mathrm{Nb}}$ diffraction peak towards larger d-spacing in the loading direction, as evident in Fig. 3b.

Stage III is associated with further elastic deformation of the transformed NiTi matrix and massive plastic deformation of both the matrix and the nanowires. In this stage, most NiTi in the matrix has undergone the B2-B19' transformation, as suggested by the fact that the intensity of each of the B19' peaks remained relative constant. The position of the (220) $\mathrm{Nb}$ diffraction peak became constant, implying plastic deformation, and the (001) B19'-NiTi $_{\text {Bi }}$ peak gradually shifted towards higher d-spacing values, implying elastic deformation, as seen in Fig. 3b.

To assess phase stresses on the NiTi matrix and on the Nb nanowires and load partitioning between the two during the process of deformation, several parameters are determined and plotted in Fig. 4, including the lattice strain of each phase, calculated stress of each phase,

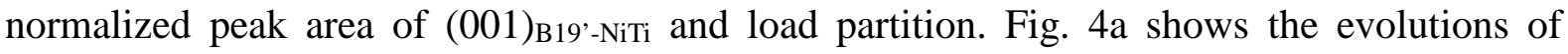
lattice strains of $(110)_{\mathrm{Nb}},(211)_{\mathrm{B} 2-\mathrm{NiTi}}$ and (001) B19'-NiTi as functions of the applied strain in the direction of loading. The lattice strain was normalized by the initial state (zero applied stress) and calculated as $\varepsilon=\left(\mathrm{d}_{\mathrm{hkl}}-\mathrm{d}_{\mathrm{hkl}}{ }^{0}\right) / \mathrm{d}_{\mathrm{hkl}}{ }^{0}$, in which $\mathrm{d}_{\mathrm{hkl}}$ is the $d$-spacing of the corresponding plane at a given applied strain, $\mathrm{d}_{h k l}{ }^{0}$ is the $d$-spacing at zero applied stress. For B19'-NiTi, since it is induced by stress, its $\mathrm{d}_{\mathrm{hkl}}{ }^{0}$ under initial state cannot be determined. For this phase standard $\mathrm{X}$-ray diffraction data from the literature is used [21]. It is seen that in stage I the lattice strains of $(110)_{\mathrm{Nb}}$ and (211) $)_{\mathrm{B} 2-\mathrm{NiTi}}$ increased linearly with the applied strain, implying linear increase of stress on both the NiTi matrix and the Nb nanowires as per the Hooke's law of elasticity. In stage II, the increase of (211)B2-NiTi lattice strain became slower, apparently due to the commencement of stress induced martensitic transformation. 

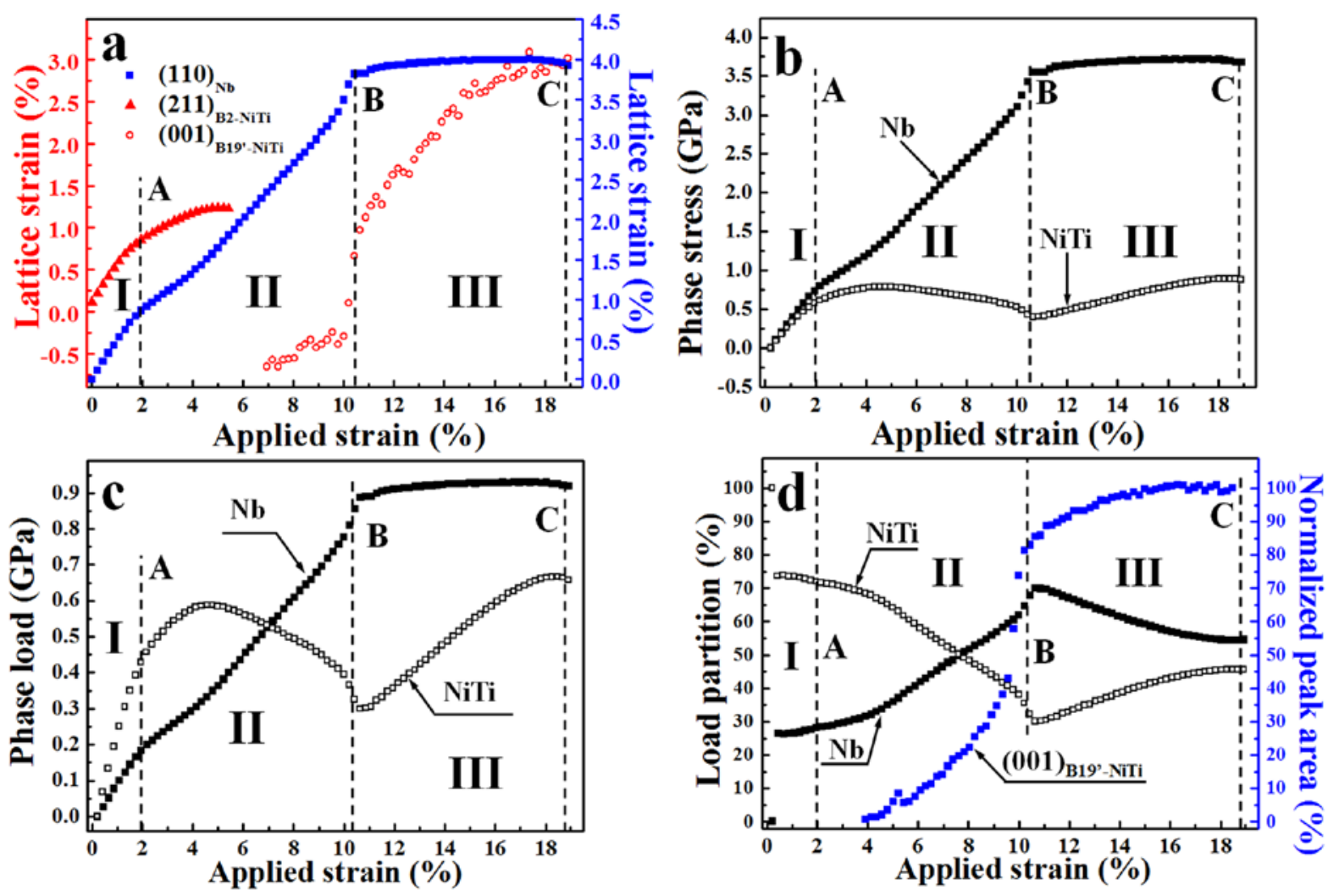

Fig. 4. Load partition between the NiTi matrix and the Nb nanowires of the composite during tensile loading. a: the lattice strain evolutions of $(110)_{\mathrm{Nb}}$, (211) $)_{\mathrm{B} 2-\mathrm{NiTi}}$ and (001) B19'-NiTi; ${ }$ b: evolution of phase stresses on Nb nanowires and NiTi matrix; c: evolution of the phase load carried by the $\mathrm{Nb}$ nanowires and the NiTi matrix; d: load partitioning between the $\mathrm{Nb}$ nanowires and the NiTi matrix and the relative peak area evolution of (001) B19'-NiTi $^{\prime}$ indicating the stress induced martensitic transformation.

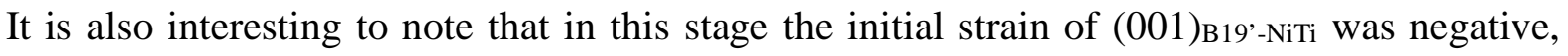
indicating that the d-spacing of newly formed B19' phase was less than the value in the database. This is attributed to the constraints of the $\mathrm{Nb}$ nanowires and surrounding $\mathrm{B} 2$ matrix. The mechanism is explained as following. At the beginning of the beginning of the stress-induced martensitic transformation, the B19' martensite formed locally has a discrete elongation much larger than elastic elongations of the adjacent $\mathrm{Nb}$ nanowires and the surrounding B2 austenite [22, 23]. This implies that the newly formed martensite will be under compression due to the constraints of the surrounding matrix. For the same reason, the elastic strains of the $\mathrm{Nb}$ nanoweires are also expected to increase more rapidly with increasing global strain. This is evident in Figure $4 \mathrm{a}$ at above $5 \%$ global strain. This explanation also implies that the effect (of negative strain) will disappear once the surrounding austenite is all consumed and the $\mathrm{Nb}$ nanowires are deformed to elastic strains of similar magnitude to the transformation strain. This is also consistent with the observation that the negative lattice strain of the martensite gradually reduced to zero and then quickly increased after the end of the transformation, as seen in Fig. 4a. 
During this stage the (001) B19'-NiTi strain increased only mildly while the matrix continued in the $\mathrm{B} 2 \rightarrow \mathrm{B} 19$ ' transformation, and the $(110)_{\mathrm{Nb}}$ lattice strain increased continuously to a maximum of $4.0 \%$. Finally in stage III, the lattice strain of the Nb nanowires remained practically constant, implying that the $\mathrm{Nb}$ nanowires had reached their yield limit (at $4.0 \%$ elastic strain) and commenced pure plastic deformation. On the other hand, the lattice strain of (001) B19'-NiTi increased rapidly at the transition from stage II to stage III, signaling the completion of the B2 $\rightarrow \mathrm{B} 19$ ' transformation, then increased continuously from $1.0 \%$ to $3.0 \%$, implying further elastic deformation of the stress-induced B19' martensite (although detwinning and reorientation of B19' martensite were also involved).

In elastic regime, various phases in the wire mainly experience one stress component (along the axial direction). When transformation starts, transverse stress and shear stress components arises in NiTi matrix, thus the calculation for strain and corresponding stress in NiTi matrix along axial direction becomes rather complex. The $\mathrm{Nb}$ nanowires, on the other hand, are strongly textured and the texture changed little during phase transformation of the NiTi matrix. Thus it is rather convenient to calculate the stress carried by nanowires from the lattice strain and the elastic modulus of Nb (110). The stress carried by NiTi matrix can thus be calculated. Meanwhile, one should recognize that the axial lattice strain of the nanowires was composed of the strain imposed by axial applied stress and the strain component along axial direction which was imposed by surrounding NiTi matrix both of which were ultimately transferred to load sharing on nanowires.

The stress on the $\mathrm{Nb}$ nanowires can be calculated directly from their lattice strain, as $\sigma_{\mathrm{Nb}}=\varepsilon_{\mathrm{Nb}} E_{\mathrm{Nb}}$. As is shown in Fig. 2, the Nb nanowires exhibit strong $<110>$ texture in the loading direction (with a magnitude of m.r.d as large as 59). Thus it is reasonable to calculate the stress on the $\mathrm{Nb}$ nanowires using the elastic modulus of $\mathrm{Nb}$ in $<110>$ crystallographic orientations, as $\sigma_{\mathrm{Nb}}=\varepsilon_{\mathrm{Nb}<110}>E_{\mathrm{Nb}<110}$, instead of that of the bulk Nb. The contribution of the $\mathrm{Nb}$ nanowires and the NiTi matrix to the total applied load is defined as phase load, as $\sigma_{\text {appl }}(x)$ $=\sigma_{x} v_{x}$, where $v_{\mathrm{x}}$ is the volume fraction of component $x$ and $\sigma_{x}$ is the stress on component $x$ in the composite. Using $E_{\mathrm{Nb}<110>} \approx 93 \mathrm{GPa}$ as the elastic modulus of the Nb nanowires along $<110>$ direction [24] and knowing the volume fraction of the $\mathrm{Nb}$ nanowires in the composite, it is easy to determine $\sigma_{\mathrm{Nb}}$ and $\sigma_{\text {appl }}(\mathrm{Nb})$. Also given that $\sigma_{\text {appl }}=\sigma_{\text {appl }}(\mathrm{NiTi})+\sigma_{\text {appl }}(\mathrm{Nb})$ and that $v_{\mathrm{Nb}}+v_{\mathrm{NiTi}}=1, \sigma_{\mathrm{appl}}(\mathrm{NiTi})$ and $\sigma_{\mathrm{NiTi}}$ can also be computed.

Fig. 4b shows the evolution of the phase stresses on the Nb nanowires and the NiTi matrix ( $\sigma_{\mathrm{Nb}}$ and $\left.\sigma_{\mathrm{NiTi}}\right)$, and Fig. 4c shows the evolution of the phase loads ( $\sigma_{\mathrm{appl}}(\mathrm{Nb})$ and $\sigma_{\mathrm{appl}}(\mathrm{NiTi})$ ), as functions of the applied strain. Both $\sigma_{\mathrm{Nb}}$ and $\sigma_{\mathrm{NiTi}}$ increased linearly in stage I. The stress on NiTi is slightly lower than that on $\mathrm{Nb}$, reflecting the relatively smaller elastic modulus of NiTi (( $E_{B 2-N i T i}=70 \sim 85$ GPa as measured by in-situ high energy x-ray diffractions) $[25,26]$ ) compared to that of $\mathrm{Nb}$ (93 GPa). The phase load shown in Fig. 3c indicates that the NiTi matrix was carrying more load than $\mathrm{Nb}$ nanowires, apparently due to its higher volume fraction in the composite. Since NiTi is the continuous matrix of the composite, the apparent modulus of NiTi can also be calculated from $E_{\mathrm{NiTi}}=\sigma_{\text {appl }}(\mathrm{NiTi}) /\left(\varepsilon_{\mathrm{NiTi}} v_{\mathrm{NiTi}}\right)=37.3 \mathrm{GPa}$, when $\varepsilon_{\mathrm{NiTi}}=\varepsilon_{\text {appl }}$. This value is calculated under the assumption that $\varepsilon_{\mathrm{NiTi}}=\varepsilon_{\mathrm{appl}}$, smaller than those 
determined by other in-situ diffraction measurements. This is because a fraction of inelastic deformation of NiTi matrix is also involved in Stage I, thus the $\mathrm{E}_{\mathrm{NiTi}}$ is more close to apparent modulus which is consistent with many reported values of apparent modulus of $\mathrm{NiTi}$ measured from global stress-strain relationships in tensile tests [27-29], rather than elastic modulus calculated from stress and lattice strain.

In stage II, $\sigma_{\mathrm{NiTi}}$ remained approximately at the same level at 0.5 0.6 GPa, with a mild increase and then a decrease during the process of deformation, whilst $\sigma_{\mathrm{Nb}}$ increased continuously, reaching a maximum of $3.5 \mathrm{GPa}$ at $10.5 \%$ of the applied strain. This demonstrates that the load was transferred from the NiTi matrix to the $\mathrm{Nb}$ nanowires when NiTi underwent stress induced martensitic transformation. It is seen in Fig. 4c that due to the load transfer from the NiTi matrix to the Nb nanowires, the phase load shared by the NiTi matrix became lower than that carried by the Nb nanowires by the end of stage II, in spite of its higher volume fraction.

In stage III, $\sigma_{N b}$ remained unchanged (Fig. 4b), implying pure plastic deformation with negligible strain hardening of the $\mathrm{Nb}$ nanwires. In contrast, $\sigma_{\mathrm{NiTi}}$ increased, reflecting the elastic deformation of the stress-induced B19' martensite. It is noted that the rate of increase of $\sigma_{\mathrm{NiTi}}$ in this stage is lower than that in stage I. This implies that the deformation of the NiTi matrix is a mixture of elastic deformation, plastic deformation and possibly further detwinning of the stress-induced B19' martensite in this post-transformation stage, consistent with previous studies [30,31]. The constant stress on the Nb nanowires implies that the increment of the applied stress all came from the contribution of the increasing load carried by the NiTi matrix, as more explicitly reflected by the phase load shown in Fig. 4c.

To assess the load sharing between the two components during the process of deformation, we define $\sigma_{\text {appl }}(x) / \sigma_{\text {appl }}$ as load partitioning on each phase [32]. Fig. $4 d$ shows the evolution of load partitioning of the NiTi matrix and the Nb nanowires. Also shown in the figure is the

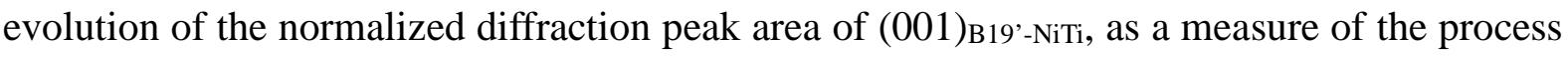
of the $\mathrm{B} 2 \rightarrow \mathrm{B} 19^{\prime}$ transformation, which is calculated by integrating the 2-D diffraction patterns from $0^{\circ}$ to $360^{\circ}$. It is seen that in stage $\mathrm{I}$, the load partitioning between the matrix and the nanowires is relatively constant, conforming to the law of mixture. The NiTi matrix carried about $73 \%$ of the applied load, which is consistent with its high volume fraction of $\sim 75 \%$. In stage II where the B2 $\rightarrow$ B19' martensitic transformation in the NiTi matrix was induced, the applied load started to transfer from the NiTi matrix to the $\mathrm{Nb}$ nanowires. The load partitioning on the nanowires rapidly increased from $28 \%$ to $70 \%$, whereas the load carried by the matrix decreased from $72 \%$ to $30 \%$. In stage III where the $\mathrm{Nb}$ nanowires experienced nearly pure plastic deformation whereas B19'-NiTi matrix experienced elastic-plastic deformation, the load partitioning of the $\mathrm{Nb}$ nanowires decreased and that of the NiTi matrix increased. The load partitioning on $\mathrm{NiTi}$ and $\mathrm{Nb}$ finally reached steady values of $45 \%$ and $55 \%$ respectively, implying that both phases have entered pure plasticity regime. It is interesting to note that the final load sharing between $\mathrm{NiTi}$ and $\mathrm{Nb}$ nanowires is different from the volume fractions of the two components. This is obviously related to the additional inelastic strain of the NiTi matrix due to its stress-induced martensitic transformation. 
The cause of load transfer between the NiTi matrix and the Nb nanowires is the change of relative stiffness of the two components during the process of deformation, i.e., the stress-induced martensitic transformation of the NiTi matrix and the plastic yielding of the $\mathrm{Nb}$ nanowires. Upon loading, the NiTi matrix undergoes SIMT with a nil stress-strain modulus whilst the $\mathrm{Nb}$ nanowires continue to deform elastically. After the completion of the SIMT, the NiTi matrix stiffens up rapidly to resume in elastic deformation mode of its stress-induced martensite whilst the $\mathrm{Nb}$ nanowires commence plastic deformation with minimum strain hardening. The changes in relative stiffness between the two components, which imply changes in load carrying capacity, whilst maintaining the same strain cause transfer of load from one component to the other. Fig. 5 shows a schematic of the mechanism of load transfer.
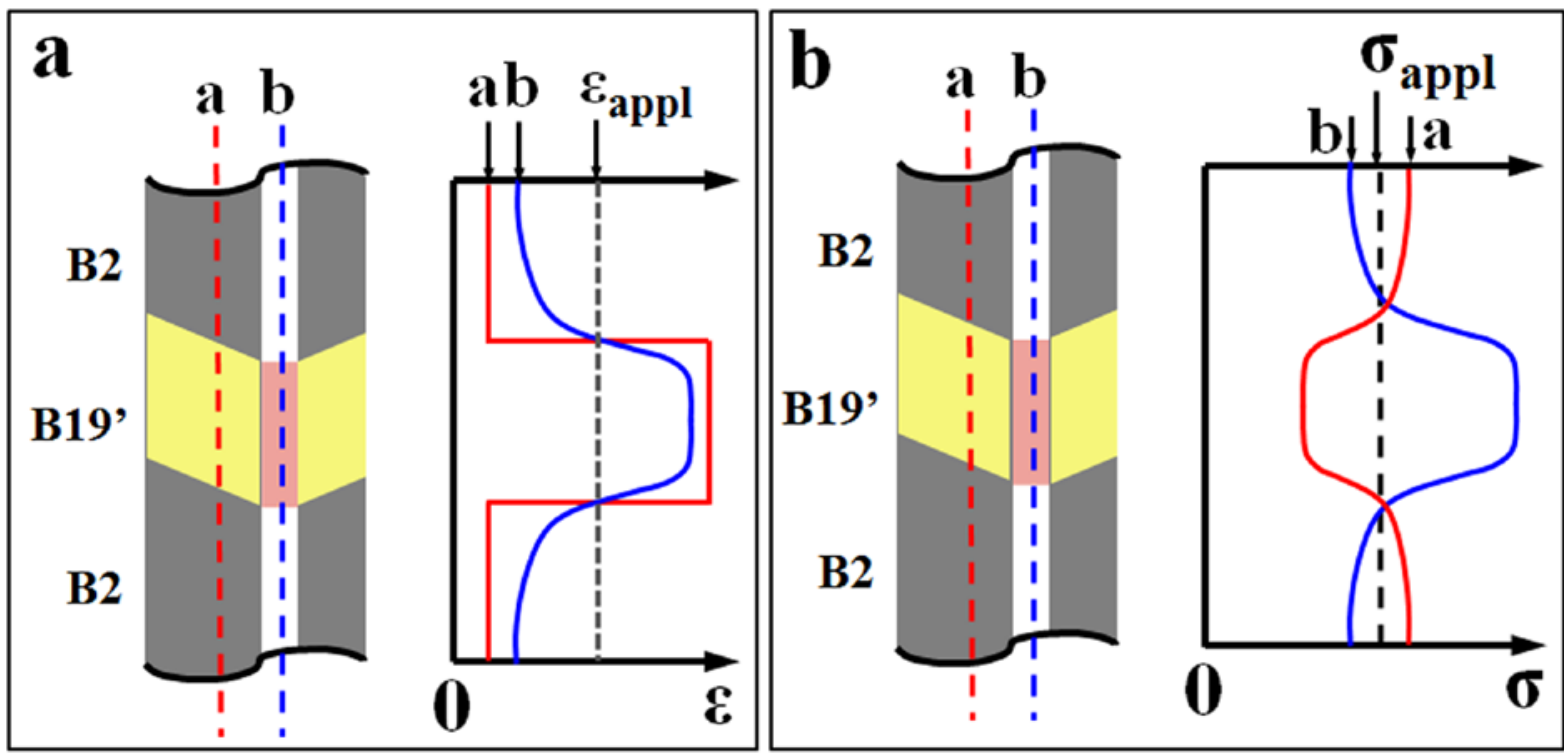

Fig. 5. Schematic of load transfer between NiTi matrix and Nb nanowires. a: the strain matching between NiTi matrix and Nb nanowire in which part of the matrix has transformed to B19' phase; b: the stress partition between NiTi matrix and Nb nanowire corresponding to a.

Fig. $5 \mathrm{a}$ is the strain distribution in the NiTi matrix and an embedded Nb nanowire during SIMT. Part of the NiTi matrix has transformed to the B19' phase. The stress-induced martensite has a discrete strain of $6 \sim 8 \%$ [28] in the direction of loading whereas the remaining austenite is still under elastic loading at $\sim 1.2 \%$ lattice strain (Fig. 4a), giving a discrete, inhomogeneous strain distribution along the length of the composite wire as indicated as line "a" and an average strain complying with the applied strain $\left(\varepsilon_{\text {appl }}\right)$. The elastic strain in the $\mathrm{Nb}$ nanowire would have naturally been $\varepsilon_{\text {appl }}$ if unconstrained. However, when constrained by the NiTi matrix, it has a distribution as indicated by line "b", i.e., under the constraint of NiTi, the embedded nanowire adjacent to the B19' phase has to deform to considerable strains trying to match the shear strain of the adjacent martensite. This means that in the B19' region, the NiTi is compressed by the $\mathrm{Nb}$ and the $\mathrm{Nb}$ is stretched by the NiTi. In the $\mathrm{B} 2$ region, the situation reverses. Fig. $5 \mathrm{~b}$ is the corresponding stress distributions in 
$\mathrm{NiTi}$ and $\mathrm{Nb}$. In the B19' region, given that the $\mathrm{NiTi}$ is under compression by the $\mathrm{Nb}$, the B19' NiTi has a stress below the applied stress whereas the Nb has a stress higher than the applied stress. In the B2 region, the reverse occurs: the B2 NiTi has a stress higher than the applied stress whereas the $\mathrm{Nb}$ has a stress lower than the applied stress. In fact, compressive elastic strains of the newly formed B19' martensite has been detected, as evident in Fig. 4a (in stage II). This is also consistent with other studies reported in the literature. Young et al. reported that within the transition zone where B2 phase and B19' phase coexist, the lattice strain of (001) B19'-NiTi $^{\prime}$ in the loading direction is negative [33].

Load transfer during deformation between the matrix and the embedded nanowires was also reported for a multi-scale filamentary $\mathrm{Cu}-\mathrm{Nb}$ composite system, which consists of a $\mathrm{Cu}$ matrix, fine $\mathrm{Cu}$ channels and $\mathrm{Nb}$ nanotubes $[13,34]$. Thilly et al. reported that the external load was transferred from the $\mathrm{Cu}$ matrix to $\mathrm{Nb}$ nanotubes when the soft $\mathrm{Cu}$ matrix yielded whilst fine $\mathrm{Nb}$ nanotubes continued their elastic deformation. In their work, the maximum elastic strain of the $\mathrm{Nb}$ nanotubes was $1.9 \%$. At a volume fraction of $20.8 \%$, these nanotubes carried a load partition of 35\%. The Cu-Nb system successfully combined high conductivity with high strength through ingenious design. Considering that the maximum elastic strain achieved is apparently smaller than what may be expected from sub-micrometer scale metallic nanostructures [13], the load carrying capacity of Nb nanotubes in copper matrix is far from being fully explored.

The NiTi-Nb composite system studied here is different from the $\mathrm{Cu}-\mathrm{Nb}$ system in that the phase transforming NiTi matrix undergoes stress induced martensitic transformation during deformation. The elongation of the matrix is caused by uniform lattice distortion instead of dislocation movement, which is inhomogeneous and highly localized at the atomic level. The uniform lattice distortion of the transformation of the NiTi matrix ensures efficient load transfer between the matrix and the nanowires during deformation, thus enabling the achievement of higher elastic strains in the nanowires, which in turn contributes to higher load sharing of the nanowires and higher overall strength of the composite. As demonstrated in this work, the maximum elastic strain achieved in the $\mathrm{Nb}$ nanowires is $4.0 \%$ and the maximum load partition is $70 \%$, with a similar volume fraction to that of the $\mathrm{Cu}-\mathrm{Nb}$ composite reported in [34]. This demonstrates that the NiTi shape memory alloy is a more suitable matrix for nanowire reinforced filament composites.

\section{Conclusions}

This study demonstrates that in a filamentary composite system composed of a phase transforming matrix and elastic nanowires, in this particular case a NiTi matrix and $\mathrm{Nb}$ nanowires, significant load transfer occurs from the more compliant component (NiTi matrix) to the more resilient component ( $\mathrm{Nb}$ nanowires) during the stress-induce martensite transformation of the matrix upon loading. In the deformation stage after the phase transformation in the NiTi matrix, reverse load transfer occurs from the plastically deforming $\mathrm{Nb}$ nanowires (more compliant) to the elastically deforming B19' martensite. Due to their high strength, the $\mathrm{Nb}$ nanowires with $25 \%$ in volume fraction are able to carry up to $70 \%$ of the total applied load. This effective load transfer within the composite provides a feasible 
mechanism for the utilization of the exceptional mechanical properties of nanowires. This study offers an in-depth understanding of the load transfer behavior of a nano-reinforcement embedded in a phase transforming matrix composite, which should have important significance in designing phase transforming composites with superior mechanical properties.

\section{Acknowledgement}

This work is supported by the National Natural Science Foundation of China (NSFC) in key program project (51231008), Australian Research Council (Grant No. DP140103805), the National 973 Programs of China (2012CB619403) and the Key Project of Chinese Ministry of Education (313055).

\section{References}

[1] Y. Lu, J. Song, J. Huang, J. Lou, Nano Res., 4 (2011) 1261-1267.

[2] G. Richter, K. Hillerich, D.S. Gianola, R. Monig, O. Kraft, C.A. Volkert, Nano Lett. , 9 (2009) 3048-3052.

[3] Y. Yue, P. Liu, Z. Zhang, X. Han, E. Ma, Nano Lett. , 11 (2011) 3151-3155.

[4] S. Wang, Y. He, H. Huang, J. Zou, G.J. Auchterlonie, L. Hou, B. Huang, Nanotechnology, 24 (2013) 285703.

[5] L.T. Ngo, D. Almécija, J.E. Sader, B. Daly, N. Petkov, J.D. Holmes, D. Erts, J.J. Boland, Nano Lett. , 6 (2006) 2964-2968.

[6] D. Qian, E.C. Dickey, R. Andrews, T. Rantell, Appl. Phys. Lett. , 76 (2000) 2868-2870.

[7] S.R.C. Vivekchand, U. Ramamurty, C.N.R. Rao, Nanotechnology, 17 (2006) S344.

[8] S. Ohsaki, K. Yamazaki, K. Hono, Scr. Mater. , 48 (2003) 1569-1574.

[9] D. Raabe, D. Mattissen, Acta Mater. , 46 (1998) 5973-5984.

[10] V. Vidal, L. Thilly, F. Lecouturier, P.-O. Renault, Scr. Mater. , 57 (2007) 245-248.

[11] L. Thilly, P.O. Renault, V. Vidal, F. Lecouturier, S. Van Petegem, U. Stuhr, H. Van Swygenhoven, Appl. Phys. Lett. , 88 (2006) 191906.

[12] V. Vidal, L. Thilly, S. Vanpetegem, U. Stuhr, F. Lecouturier, P. Renault, H. Vanswygenhoven, Scr. Mater. , 60 (2009) 171-174.

[13] L. Thilly, S.V. Petegem, P.-O. Renault, F. Lecouturier, V. Vidal, B. Schmitt, H.V. Swygenhoven, Acta Mater. , 57 (2009) 3157-3169.

[14] Paul Podsiadlo, Amit K. Kaushik, Ellen M. Arruda, Anthony M. Waas, Bong Sup Shim, Jiadi Xu,Himabindu Nandivada, Benjamin G. Pumplin, Joerg Lahann, Ayyalusamy Ramamoorthy, Nicholas A. Kotov., Science 318, 80 (2007) 80-83.

[15] Y. Dzenis, Science, 319 (2008) 419-420.

[16] U.K. J. N. Coleman, Y. K. Gun'ko, Adv. Mater 18, 6 (2006) 689-706.

[17] S. Hao, L. Cui, D. Jiang, X. Han, Y. Ren, J. Jiang, Y. Liu, Z. Liu, S. Mao, Y. Wang, Y. Li, X. Ren, X. Ding, S. Wang, C. Yu, X. Shi, M. Du, F. Yang, Y. Zheng, Z. Zhang, X. Li, D.E. Brown, J. Li, Science, 339 (2013) 1191-1194.

[18] A.P. Hammersley, FIT2D V9.129 reference manual V3.1, ESRF Internal Report, (1998).

[19] L. Lutterotti, S. Matthies, H.-R. Wenk, A. S. Schulz, and J. W. Richardson, Jr, J. Appl. Phys, 81 (1997) 594-600.

[20] M. Piao, Miyazaki, S., Otsuka, K., Nishida, N., Japan Institute of Metals, 33 (1992) 337.

[21] Y. Kudoh, M. Tokonami, S. Miyazaki, K. Otsuka, Acta Metallurgica, 33 (1985) 2049-2056.

[22] S. Miyazaki, K. Otsuka, Y. Suzuki, Scripta Metallurgica, 15 (1981) 287-292.

[23] X. Zhang, H. Sehitoglu, Mater. Sci. Eng., A 374 (2004) 292-302.

[24] G.N. Nadezhdin, I.S. Malashenko, A.L. Kuporev, Strength Mater. , 1 (1969) 385-389.

[25] O. Benafan, R.D. Noebe, S.A. Padula Ii, A. Garg, B. Clausen, S. Vogel, R. Vaidyanathan, International Journal 
of Plasticity, 51 (2013) 103-121.

[26] Y. Liu, H. Yang, Mater. Sci. Eng., A, 260 (1999) 240-245.

[27] Y. Liu, H. Xiang, Journal of Alloys and Compounds, 270 (1998) 154-159.

[28] C. Greiner, S.M. Oppenheimer, D.C. Dunand, Acta biomaterialia, 1 (2005) 705-716.

[29] J.-E. Bidaux, L. Bataillard, J.-A. Maanson, R. Gotthardt, Le Journal de Physique IV, 3 (1993) C7-561-C567-564.

[30] Y. Liu, Y. Liu, J. Van Humbeeck, Acta Mater. , 47 (1998) 199-209.

[31] G. Tan, Y. Liu, P. Sittner, M. Saunders, Scr. Mater. , 50 (2004) 193-198.

[32] S.J. Hao, D.Q. Jiang, L.S. Cui, Y.D. Wang, X.B. Shi, Z.H. Nie, D.E. Brown, Y. Ren, Appl. Phys. Lett. , 99 (2011) 084103-084103.

[33] M.L. Young, M.F.X. Wagner, J. Frenzel, W.W. Schmahl, G. Eggeler, Acta Mater. , 58 (2010) 2344-2354.

[34] L. Thilly, P.O. Renault, S. Van Petegem, S. Brandstetter, B. Schmitt, H. Van Swygenhoven, V. Vidal, F. Lecouturier, Appl. Phys. Lett. , 90 (2007) 241907. 